\title{
Telementoring for communication between residents and faculty physicians: Results from a survey on attitudes and perceptions in an Academic Tertiary Urology Referral Department in Italy
}

\author{
Vincenzo Mirone ${ }^{1}$, Massimiliano Creta ${ }^{1}$, Marco Capece ${ }^{1}$, Giuseppe Celentano ${ }^{1}$, Gianluigi Califano ${ }^{1}$, \\ Claudia Collà Ruvolo ${ }^{1}$, Lorenzo Spirito ${ }^{1}$, Giovanni Maria Fusco ${ }^{1}$, Luigi Cirillo ${ }^{1}$, Nicola Longo ${ }^{1}$, \\ Ferdinando Fusco $^{2}$, Claudia Mirone ${ }^{3}$, Roberto La Rocca ${ }^{1}$, Luigi Napolitano ${ }^{1}$ \\ ${ }^{1}$ Department of Neurosciences, Reproductive Sciences and Odontostomatology, University of Naples "Federico II", Naples, Italy; \\ 2 Department of Woman, Child and General and Specialized Surgery, Urology Unit, University of Campania "Luigi Vanvitelli"" \\ Naples, Italy; \\ ${ }^{3}$ Multidisciplinary Department of Medical, Surgical and Dental Sciences, University of Campania "Luigi Vanvitelli”, Naples, Italy.
}

\begin{abstract}
Summary Introduction: Telemedicine has been adopted successfully in various urological scenarios.

The aim of the present study was to explore attitudes and perceptions by urology residents toward the use of telementoring in the context of residents-faculty physicians communication for patient-related care.

Methods: An online survey consisting of 19 multiple choice questions was designed including three sections: respondents' demographics, attitudes and perceptions towards the use of telementoring. Invitations to participate in this anonymous survey were e-mailed to urology residents at University of Naples Federico II. Results: In total 60 responses were received (participation rate $86 \%$ ). The frequency of telementoring use was described as occasional, frequent, very frequent, and rare by $51,3 \%, 41.0 \%, 5,1 \%$, and $2,6 \%$ of respondents, respectively. WhatsApp messenger was used by $89.5 \%$ of respondents and photos were the most common type of media content shared $(73.7 \%)$. Most of respondents declared a moderate and a strong agreement with respect to the utility of telementoring in improving the communication in relation to the interpretation of clinical, radiological, endoscopic, and functional findings. Overall, $78 \%$ of participants individuated risks of information flow distortions and misinterpretations as the major limit of telementoring.

Conclusions: The use of telementoring is widespread and perceived as useful by urology residents in the context of residentsfaculty physicians communication in multiple settings of patientrelated care.
\end{abstract}

KEY WORDS: Telementoring; Telemedicine; Urology; Bladder cancer; Patient-related care; Survey; Pandemic; COVID-19: Medical informatics.

Submitted 15 July 2021; Accepted 2 August 2021

\section{INTRODUCTION}

Telehealth, defined as the use of information technology and telecommunications to provide access to health assessment, consultation, diagnosis, intervention, supervision and information across distance, represents a rapidly evolving field of medicine (1). Telemedicine is con- sidered to be under the umbrella of telehealth and refers specifically to remote clinical services (2). Remote care provides the advantage of reducing the use of resources in health centers, improving access to care, and minimizing the risk of direct transmission of the infectious agent (3). The adoption of telemedicine was first described in the 1950's when a Nebraska psychiatrist connected to a prison over 150 miles through a closed-circuit television to provide mental health services (4). In recent years, with the advancement of mobile technologies, telemedicine is more accessible than ever before. In the USA the percentage of US hospitals that connect with patients through the use of video and other technology has increased from 35\% in 2010 to $76 \%$ in 2017 (1).

Available literature indicates that telemedicine has been adopted successfully in patients with common clinical urological conditions, including prostate cancer, uncomplicated urinary stones, uncomplicated urinary infections, urinary incontinence, or pelvic organ prolapse and hematuria (5). Moreover, literature on the use of telemedicine in medical education, called telementoring, is increasing and results underline the facilitation of the learning process while increasing motivation and enabling instant communication and discussion at a distance due to the possibility of sharing multimedia contents (6). The aim of the present study was to explore attitudes and perceptions by urology residents toward the use of telementoring in the context of residents- faculty physicians communication for patient-related care in a large academic tertiary urology referral department in Italy.

\section{Methods}

Questionnaire

An online survey consisting of 19 multiple choice questions (formulated in Italian with the aim of increasing the response rate) was designed using the Google Form application included in the Google Drive office suite 
(Google LLC). The questionnaire was composed of three sections: a first one to assess respondents' demographics (age, sex, year of residency) a second one to evaluate their attitudes, and a third to evaluate perceptions.

Questions about attitudes toward use of telementoring during the residency program and in the context of resident to faculty physicians communication for patientrelated care investigated the following settings: frequency, duration, and temporal trend of telementoring adoption, tools adopted (telephone call, e-mails, WhatsApp, others) clinical context in which telementoring was used (ward, ambulatory care, referral activities), type of findings whose interpretation made use of telementoring (findings from physical examination, findings from radiological imaging, findings from endoscopy, findings from functional investigations), type of shared data (text, video, audio, photos), content of multimedia data shared (reports, radiological images, drainages, findings from physical examination, endoscopic findings, intraoperative findings).

Questions about perceptions investigated the perceived usefulness of telementoring in improving resident to faculty physicians communication for patient-related care in various clinical settings (ward, ambulatory care, referral activities) and in relation to various clinical and instrumental findings (findings from physical examination, endoscopy, radiological imaging, functional investigations). Respondents were invited to "strongly agree", "moderately agree", "slightly agree", "strongly disagree", "moderately disagree", "slightly disagree" with a series of statements about perceptions. A question was designed to investigate how often the use of telementoring was able to change the diagnostic and/or therapeutic decision-making process.

Question about the perceived limits of telementoring investigated: medico-legal issues, risk of distortion of the information flow, risk of receiving incomplete data, risk of providing incomplete data.

Some questions required a single answer while others gave the respondents the choice to select as many answers as they felt appropriate.

\section{Data collection}

Invitations to participate in this anonymous survey were e-mailed on 1 February 2021 to current urology residents and to urology residents who attended the urology residence program in the last three years at University of Naples Federico II and who gave the approval to the use of their e-mail address. For those who had not completed the survey, four follow-up reminder e-mail invitations were sent over the following 2 weeks. The survey was closed on 28 February 2021. All respondents had to fully complete the questionnaire before submission since all questions were flagged as mandatory. After submission, users could not review neither amend their answers. Both personal contact information and data collected were not accessible to third parties.

Figure 2.

\section{Data analysis}

Data were expressed as mean (Standard Deviation) and raw numbers and percentages of survey answers. Statistical analyses were two-sided using a significance level of 0.05 . All statistical analyses were performed with SPSS version 17.0 (SPSS, Inc., Chicago, IL) software.

\section{RESULTS}

In total 60 responses were received (participation rate 86\%). Fifty-seven residents (95\%) were male and $3(5 \%)$ were female. Mean age was 31.36 (3.16). Overall, 23.33\% were $1^{\text {st }}$ years residents, $16.62^{\text {nd }}$ year, $10 \% 3^{\text {rd }}$ year, $15 \%$ $4^{\text {th }}$ year, $23.3 \% 5^{\text {th }}$ year and $11.67 \%$ were specialist less than two year. The frequency of telementoring use in the context of resident to faculty physicians communication for patient-related care was described as occasional, frequent, very frequent, and rare by $51.3 \%, 41.0 \%, 5.1 \%$, and $2.6 \%$, respectively.

Figure 1 shows the setting of utilization of telementoring use by urology residents.

Figure 2 describes the percentage of utilization by residents of the different tools of telementoring in the context of resident to faculty physicians communication for patient-related care.

Figure 1.

Setting of telementoring use in the context of resident to faculty physicians communication for patient-related care and relative percentage of use in the various settings.

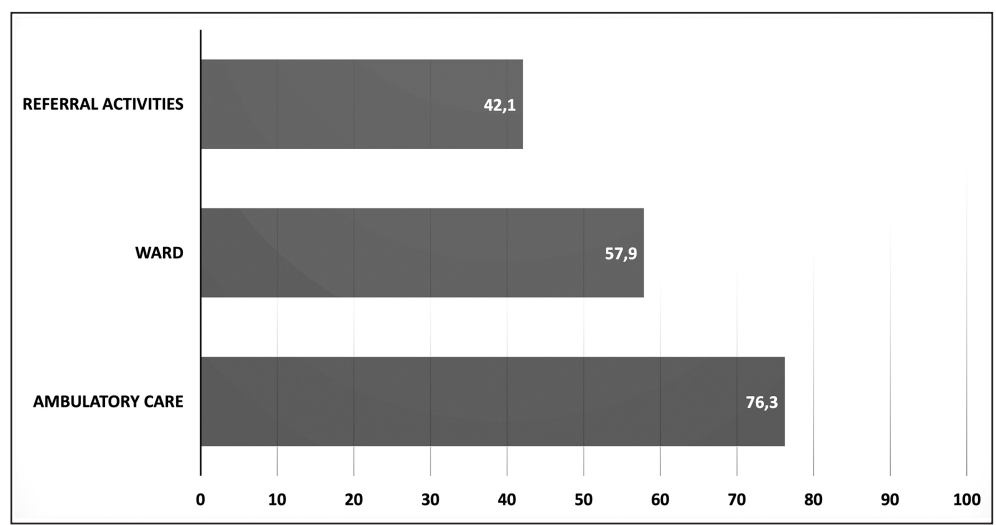

Percentage of residents using different tools of telementoring in the context of resident to faculty physicians communication for patient-related care.

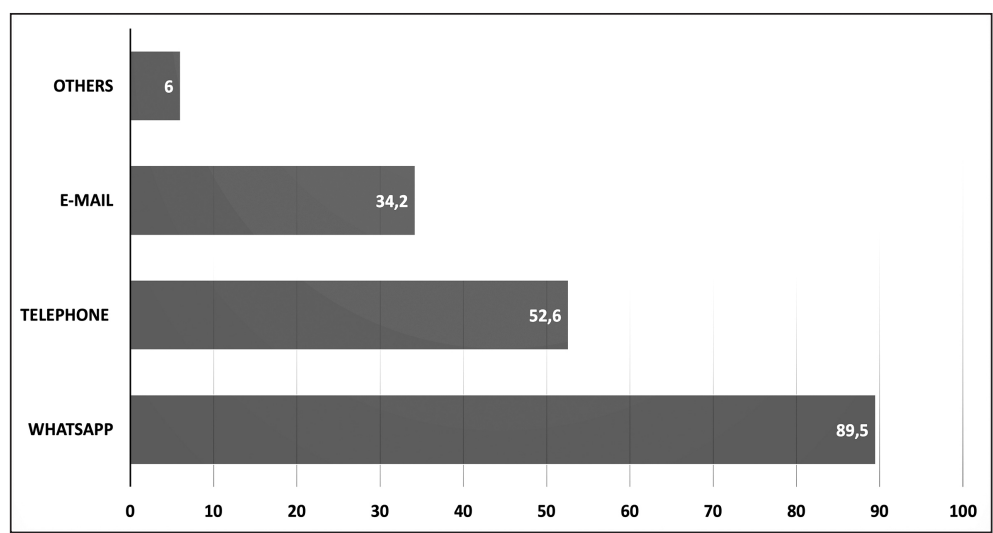


Figure 3.

Percentage of residents sharing different types of media contents in the context of resident to faculty physicians communication for patient-related care.

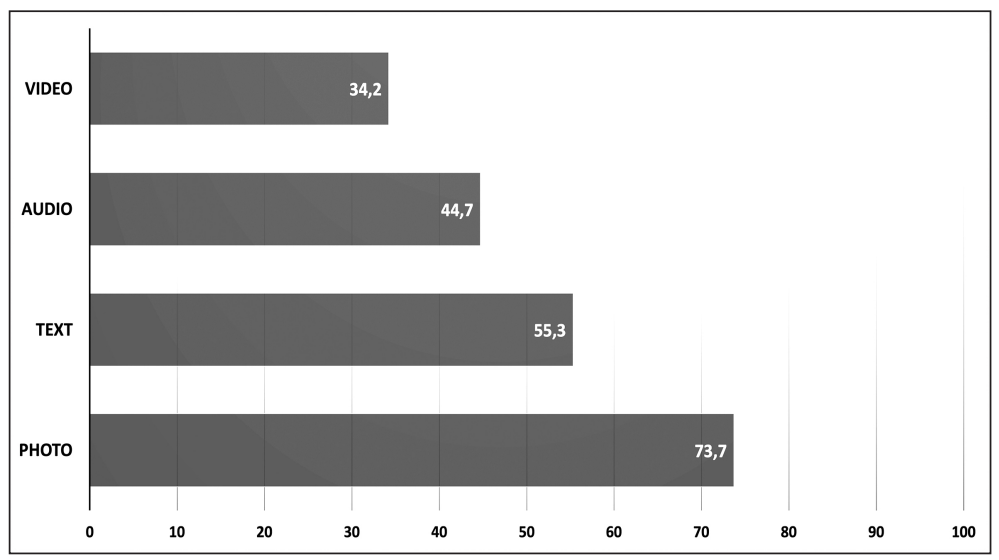

Figure 4.

Percentage of residents sharing different types of information as media contents in the context of resident to faculty physicians communication for patient-related care.

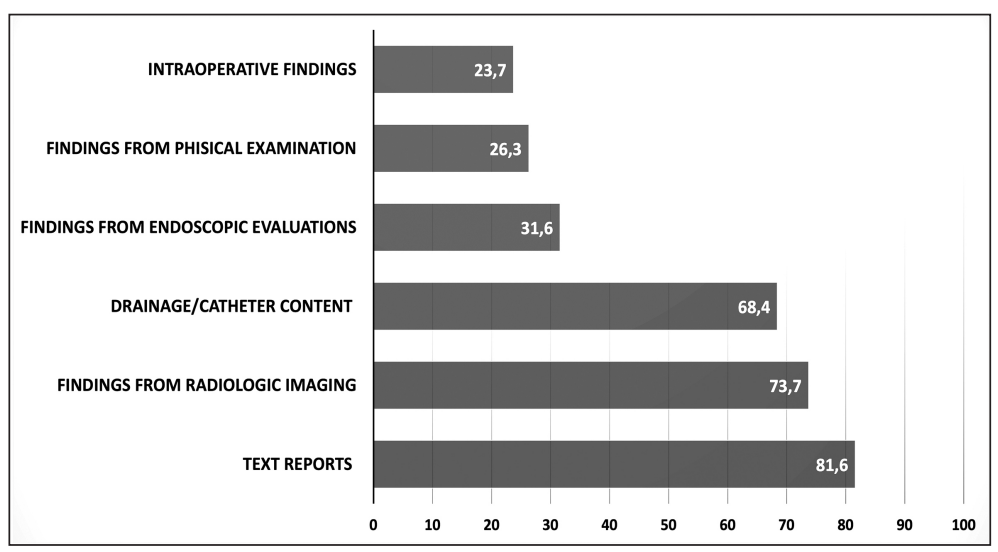

Figure 3 shows the percentage of residents sharing different types of media contents in the context of resident to faculty physicians communication for patient-related care.

Figure 4 shows the percentage of residents sharing different types of information as media contents in the context of resident to faculty physicians communication for patient-related care. Table 1 describes the perceptions about the usefulness of telementoring in improving resident to faculty physicians communication for patient-related care in various clinical settings and in relation to various clinical and instrumental findings.

Finally, regarding perception of telementoring limitation, $78 \%$ of participants individuated risks of information flow distortions and misinterpretations, $56.4 \%$ found limits for a complete information collection, $35.9 \%$ risks of flow distortions to give information, and $28.2 \%$ legal risk.

\section{Discussion}

Remote communication between members of a medical staff has always been the key element in any part of patients' care (7). Of note, poor communication was considered by the 2011 Joint Commission sentinel event report as the main root cause of delays in patient treatment and the second leading cause of operative and post-operative complications (8).

Interprofessional communication has a critical role mainly in university hospitals, where residents are involved in the management of patients. Indeed, ineffective communication between residents and attending surgeons has been reported to be a significant contributor to

\section{Table 1.}

Perceptions about the usefulness of telementoring in improving resident to faculty physicians communication for patient-related care in various clinical settings and in relation to various clinical and instrumental findings.

\begin{tabular}{|c|c|c|c|c|c|c|}
\hline & $\begin{array}{l}\text { Agree strongly } \\
\mathrm{n}(\%)\end{array}$ & $\begin{array}{l}\text { Agree moderately } \\
\text { n (\%) }\end{array}$ & $\begin{array}{l}\text { Agree slightly } \\
\mathrm{n}(\%)\end{array}$ & $\begin{array}{l}\text { Disagree slightly } \\
\quad \mathrm{n}(\%)\end{array}$ & $\begin{array}{l}\text { Disagree moderately } \\
\qquad \mathrm{n}(\%)\end{array}$ & $\begin{array}{l}\text { Disagree strongly } \\
\mathrm{n}(\%)\end{array}$ \\
\hline $\begin{array}{l}\text { Telementoring is useful in improving resident to faculty physicians } \\
\text { communication for patient-related care in the ward setting }\end{array}$ & $14(23.3)$ & $35(58.3)$ & $11(18.3)$ & $0(0)$ & $0(0)$ & $0(0)$ \\
\hline $\begin{array}{l}\text { Telementoring is useful in improving resident to faculty physicians } \\
\text { communication for patient-related care in the ambulatory setting }\end{array}$ & $14(23.3)$ & $36(60.0)$ & $8(13.3)$ & $0(0)$ & $2(3.3)$ & $0(0)$ \\
\hline $\begin{array}{l}\text { Telementoring is useful in improving resident to faculty physicians } \\
\text { communication for patient-related care in the referral activity setting }\end{array}$ & $17(28.3)$ & 32 (53.3) & $9(15.0)$ & $2(3.3)$ & $0(0)$ & $0(0)$ \\
\hline $\begin{array}{l}\text { Telementoring is useful in improving resident to faculty physicians } \\
\text { communication for patient-related care thanks to the improvement } \\
\text { of radiological imaging interpretation }\end{array}$ & $17(28.3)$ & 32 (53.3) & $8(13.3)$ & $3(5.0)$ & $0(0)$ & $0(0)$ \\
\hline $\begin{array}{l}\text { Telementoring is useful in improving resident to faculty physicians } \\
\text { communication for patient-related care thanks to the improvement } \\
\text { of endoscopic findings interpretation }\end{array}$ & $14(23.3)$ & $27(45.0)$ & $17(28.3)$ & $2(3.3)$ & $0(0)$ & $0(0)$ \\
\hline $\begin{array}{l}\text { Telementoring is useful in improving resident to faculty physicians } \\
\text { communication for patient-related care thanks to the improvement } \\
\text { of interpretation of findings from physical examination }\end{array}$ & $14(23.3)$ & $35(58.3)$ & 11 (18.3) & $0(0)$ & $0(0)$ & $0(0)$ \\
\hline $\begin{array}{l}\text { Telementoring is useful in improving resident to faculty physicians } \\
\text { communication for patient-related care thanks to the improvement } \\
\text { of functional findings interpretation }\end{array}$ & $6(10.0)$ & $30(50.0)$ & $14(23.3)$ & $10(16.6)$ & $0(0)$ & $0(0)$ \\
\hline
\end{tabular}


medical errors, patient injury, and malpractice claims (9). Verbal report via telephones has represented the traditional method of communication for years (10). However, although rapid, this method of communication can be inadequately objective and precise (10). Since the late 1990's telemedicine gained popularity and clinical photographs taken by digital cameras were transmitted as downloadable files between computers having modem and telephone link to improve communication flow (10). Initially, several technological limitations including the lack of digitalization of medical records, the inability to send images quickly, and limited Internet lines strongly limited the use of telemedicine. In recent years, advances in information technology have driven dramatic changes in several aspects of human behavior and communication. Audiovisual communication in health care supported by smartphone apps is a novel concept that is rapidly gaining interest in all areas of medicine and surgery (11). Implementation of telemedicine is currently encouraged and supported by both states and multiple medical associations worldwide (12).

To the best of our knowledge, we investigated for the first-time attitudes and perceptions about the use of telementoring in the context of resident to faculty physicians communication for patient-related care in an academic tertiary urology referral department in Italy.

Most urology residents participating in the present survey declared to utilize telementoring for patient-related care and that the use of telementoring increased over time. This finding is in line with available evidence demonstrating the expanding role of telehealth (1).

WhatsApp messenger was reported as the most commonly used tool for communication. WhatsApp is a method of sending and receiving messages to and from individuals or groups with additional features of sending images, videos, and links (13). WhatsApp has been evaluated in numerous subspecialties in both undergraduate and postgraduate settings and current available literature suggests it is an effective tool for medical learning (14). Some authors also consider WhatsApp as an effective telemedicine tool in many different fields of health care (11).

Photos followed by text messages were the most common type of data shared by the respondents in present survey. In details, the main contents of photos were medical reports followed by radiological images and pictures of drainages. Accordingly, Sener et al. suggest that WhatsApp can be used to share photos of cases of hematuria to determine the severity of cases, and thereby discriminate whether active treatment is required (15).

Urology residents are commonly involved in multiple activities characterized by increasing complexity. Most of respondents in the present survey perceived telementoring e as a useful communication tool in several aspects of patient care. Indeed, the majority of them declared a moderate and a strong agreement towards the utility of telementoring in improving resident to faculty physicians communication for patient-related care in the context of ambulatory care, ward, and urology referral consultation. In details, most declared a moderate and a strong agreement with respect to the utility of telementoring in improving the communication in relation to the interpretation of clinical, radiological, endoscopic, and function- al findings. Interestingly, a high percentage of residents declared that the use of telementoring in the context of resident to faculty physicians communication for patientrelated care contributed to change the diagnostic and/or therapeutic decision making process.

Taken together, results from the present survey underline the increasing and relevant role of telementoring as an additional tool in the context of resident to faculty physicians communication in several settings of patient-related care.

However, telementoring is still in its infancy and a number of potential drawbacks still exist. These include mainly limitations with performing comprehensive medical history and physical examination and security breaches (2). Distortion of the medical information and limits in acquiring complete information were perceived by the participants in the present survey as the major drawbacks of telementoring in the context of resident to faculty physicians communication for patient-related care.

The major limitation of this survey includes the small number of participants. Although respondents are not fully representative of the overall community of Italian urology residents, currently the School of Urology of the University of Naples Federico II represents the largest of the Southern Italy in terms of number of residents and one of the largest in Italy. Moreover, like any survey, participant responses were limited to the available choices. A further limit of the study is the lack of data about the feedback of faculty physicians. Further studies are needed to investigate the usefulness of telementoring by residents in relation to specific diseases (16).

\section{Conclusions}

Results from the present survey demonstrate that the use of telementoring is widespread and perceived as useful by urology residents in the context of resident to faculty physicians communication in multiple settings of patientrelated care and its use has increased over time. WhatsApp messenger is the most common tool adopted for remote communication with text and photos of reports and drainages being the most common data shared.

\section{REFERENCES}

1. Kichloo A, Albosta M, Dettloff K, et al. Telemedicine, the current COVID-19 pandemic and the future: a narrative review and perspectives moving forward in the USA. Fam Med Community Health. 2020; 8:e000530.

2. Gajarawala SN, Pelkowski JN. Telehealth benefits and barriers. J Nurse Pract JNP. 2021; 17:218-221.

3. Wittson CL, Affleck DC, Johnson V. Two-way television in group therapy. Ment Hosp. 1961; 12:22-3.

4. Monaghesh E, Hajizadeh A. The role of telehealth during COVID19 outbreak: a systematic review based on current evidence. BMC Public Health. 2020; 20:1193.

5. Novara G, Checcucci E, Crestani A, et al. Telehealth in Urology: a systematic review of the literature. How much can telemedicine be useful during and after the COVID-19 pandemic? Eur Urol. 2020; 78:786-811.

6. Mazzuoccolo LD, Esposito MN, Luna PC, et al. WhatsApp: A realtime tool to reduce the knowledge gap and share the best clinical 
practices in psoriasis. Telemed J E-Health Off J Am Telemed Assoc. 2019; 25:294-300.

7. Haykal T, Al-Dulaimi R, Sidahmed S, et al. Understanding the means of communication between nurses and resident physicians in the modern world: A community-based university hospital survey results. J Community Hosp Intern Med Perspect. 2020; 10:107-110.

8. Commission TJ. TJ Commission. Sentinel event data root causes by event type 2004-Third Quarter 2011. Washington, DC: The Joint Commission; 2011.

9. Senders ZJ, Aeder M, Semrau S, et al. Improving resident-toattending communication: implementing a tool to facilitate attending notification of critical patient events at a single academic institution. Am Surg. 2019; 85:663-670.

10. Wani SA, Rabah SM, AlFadil S, et al. Efficacy of communication amongst staffcmembers at plastic and reconstructive surgery section using smartphone and mobile WhatsApp. Indian J Plast Surg Off Publ Assoc Plast Surg India. 2013; 46:502-5.

11. Giordano V, Koch H, Godoy-Santos A, et al. WhatsApp messen- ger as an adjunctive tool for telemedicine: an overview. Interact J Med Res. 2017; 6:e11.

12. Becker CD, Dandy K, Gaujean M, et al. Legal perspectives on telemedicine Part 1: legal and regulatory issues. Perm J. 2019; 23:18-293.

13. Bakshi SG, Bhawalkar P. Role of WhatsApp-based discussions in improving residents' knowledge of post-operative pain management: a pilot study. Korean J Anesthesiol. 2017; 70:542-549.

14. Coleman E, O'Connor E. The role of WhatsApp ${ }^{\circledR}$ in medical education; a scoping review and instructional design model. BMC Med Educ. 2019; 19:279.

15. Sener TE, Butticè S, Sahin B, et al. WhatsApp Use in the evaluation of hematuria. Int J Med Inf. 2018; 111:17-23.

16. Ambrosini F, Di Stasio A, Mantica G, Cavallone B, Serao A. COVID-19 pandemic and uro-oncology follow-up: A "virtual" multidisciplinary team strategy and patients' satisfaction assessment. Arch Ital Urol Androl. 2020; 92:78-79..

\section{Correspondence}

Vincenzo Mirone, MD

mirone@unina.it

Massimiliano Creta, MD, PhD (Corresponding Author)

max.creta@gmail.com

Marco Capece, MD

drmarcocapece@gmail.com

Giuseppe Celentano, MD

dr.giuseppecelentano@gmail.com

Gianluigi Califano, MD

gianl.califano2@gmail.com

Claudia Collà Ruvolo, MD

c.collaruvolo@gmail.com

Lorenzo Spirito, MD

lorenzospirito@msn.com

Giovanni Maria Fusco, MD

giom.fusco@gmail.com

Luigi Cirillo, $M D$

cirilloluigi22@gmail.com

Nicola Longo, MD

nicola.longo@unina.it

Roberto La Rocca, MD

robertolarocca87@gmail.com

Luigi Napolitano, MD

luiginap89@gmail.com

Department of Neurosciences, Reproductive Sciences and

Odontostomatology, University of Naples "Federico II", 80131 Naples (Italy)

Ferdinando Fusco, MD

ferdinando-fusco@libero.it

Department of Woman, Child and General and Specialized Surgery, Urology

Unit, University of Campania "Luigi Vanvitelli"' 80131 Naples (Italy)

Claudia Mirone, MD

claudiamirone@outlook.it

Multidisciplinary Department of Medical, Surgical and Dental Sciences,

University of Campania "Luigi Vanvitelli", 80131 Naples (Italy) 